\title{
Examination of Urinary Chlorpyrifos Biomarker Concentrations and Heart Rate in a Sample of US Children and Adolescents
}

\author{
Alex LeBeau, Desirae Sutherland, Marie Bourgeois, Jeffrey Driver, Raymond Harbison \\ Center for Environmental and Occupational Risk Analysis and Management, College of Public Health, University of South \\ Florida, Tampa, Florida, USA \\ Email: alebeau@mail.usf.edu, desirae@usf.edu
}

How to cite this paper: LeBeau, A., Sutherland, D., Bourgeois, M., Driver, J. and Harbison, R. (2020) Examination of Urinary Chlorpyrifos Biomarker Concentrations and Heart Rate in a Sample of US Children and Adolescents. Occupational Diseases and Environmental Medicine, 8, 163-174.

https://doi.org/10.4236/odem.2020.84013

Received: September 20, 2020

Accepted: October 17, 2020

Published: October 20, 2020

Copyright $\odot 2020$ by author(s) and Scientific Research Publishing Inc. This work is licensed under the Creative Commons Attribution International License (CC BY 4.0).

http://creativecommons.org/licenses/by/4.0/

\begin{abstract}
Objective: The purpose of the investigation is to determine if a relationship exists between heart rate and urinary biomarkers of chronic, subacute chlorpyrifos pesticide exposure in youth. Methods: Using 2001-2002 NHANES data, a sample of 1233 children ages 6 - 18 was grouped based on detection status of 3,5,6-trichloropyridinol (TCPy) in urine. Radial pulse and brachial pulse were recorded as measures of heart rate by physicians. $T$-tests and linear regression analyses were performed to test for associations between TCPy concentrations and heart rate. Results: None of the associations between TCPy levels and heart rate outcomes were found to be significant. Nonsignificant effects in the TCPy-detected groups included a slightly reduced heart rate in girls, as well as a slightly elevated heart rate in boys when compared to the undetected controls. Neither was there any significant observable difference in heart rate due to detection status in the sample overall. Conclusions: At this time, an effect on heart rate attributable to chronic, low-level chlorpyrifos exposure in youth cannot be determined. Children and adolescents detected did not demonstrate a substantial change in pulse measures when compared to controls. It is recommended that subsequent studies examine chlorpyrifos biomarkers as they may relate to other indicators of cardiovascular health.
\end{abstract}

\section{Keywords}

Chlorpyrifos, Organophosphates, Pesticides, Acetylcholinesterase Inhibitors, TCPy, Heart Rate, Pulse, Cardiovascular, Exposure, Urinary Metabolites, Children, Youth, Adolescents 


\section{Introduction}

Organophosphates (OP) are pervasive globally due to their utility in pest control and farming [1] [2]. Potential bystander exposures may occur by inhaling spray drift from agricultural applications, or ingesting drinking water and plant foods bearing OP residues [3]. Dermal and inhalation exposure may also occur in workers during pesticide mixing, loading and application, as well as during reentry (e.g., harvesting) activities) [3]. Although chlorpyrifos is one of the most commonly applied organophosphates in agriculture, residential use was banned by the US Environmental Protection Agency (US EPA) in 2001 [2] [4] [5]. Fleas, termites, mosquitos, and roaches are vulnerable to the actions of chlorpyrifos, and its effectiveness has allowed it to remain in use despite the controversy regarding its safety [6] [7]. Current agricultural applications of chlorpyrifos include corn, broccoli, soybeans, Brussels sprouts, and fruit trees [8]. The use of chlorpyrifos also extends to non-agricultural settings including the maintenance of grassy lawns within golf courses, application on wood constructs including fencing, and mosquito control for public health [8].

Chlorpyrifos is lipophilic, in that it may be stored in fatty tissues if not eliminated from the body [7]. Oral and inhalation exposures tend to produce the highest concentrations of internalized chlorpyrifos, with dermal exposure representing a lower fraction of absorption [7]. Once absorbed, chlorpyrifos exerts an acetylcholinesterase-inhibitory effect following its conversion to the chlorpyrifos-oxon by cytochrome P450 [7] [9]. This transformation confers its ability to bind and inhibit cholinesterases [7] [10]. Chlorpyrifos-oxon can undergo hydrolysis by phosphotriesterase paraoxonase1 (PON1) converting it into a dialkyl phosphate and 3,5,6-trichloro-2-pyridinol (TCPy) destined for elimination in urine [2] [11]. Thus, TCPy is the metabolite which is commonly used as a biomarker to establish exposure to chlorpyrifos [7] [11].

There is some concern that children and fetuses in utero are vulnerable to harm from organophosphate pesticides [12]. This is owing to the lower expression of the detoxifying PON1 enzymes in young children which at insufficient levels may impair the ability to transform chlorpyrifos-oxon into an innocuous metabolite [7]. It has been asserted that a mother's PON1 genotype may afford her offspring either a degree of resistance to OP associated toxicity, or decreased resilience to OP [4] [13]. Prenatally, chlorpyrifos and chlorpyrifos-oxon have also been shown to move through the placenta to cause placental cell death [14]. Indeed, maternal blood and placental concentrations of chlorpyrifos have been found to be highly correlated [15]. Thus, an interplay between an individual's age during exposure and genetic composition is critical as it may influence a child's susceptibility to the actions of chlorpyrifos [16].

The primary mechanism of chlorpyrifos toxicity is the irreversible binding of chlorpyrifos-oxon to acetylcholinesterases (AChE) [7]. Acetylcholine is a neurotransmitter which is capable of binding to M2 muscarinic receptors in cardiac tissue [17]. AChE disable the reunion of acetylcholine and the associated recep- 
tor [17]. Thus, the potential for AChE inhibition by chlorpyrifos may permit increased binding between acetylcholine and its receptors, leading to the alteration in heart rate at toxic doses. In cases of acute chlorpyrifos poisoning, cardiac abnormalities can manifest as both bradycardia and tachycardia depending upon a variety of factors including dosage [18] [19]. Arrhythmias have also been produced in rats, particularly bradycardia at dosages of $25 \mathrm{mg} / \mathrm{kg}$ chlorpyrifos [20]. However, less is known regarding the effects of chlorpyrifos specifically on heart rate for chronic, low-level exposure in the general population. The purpose of the current analysis is to determine the effect that chlorpyrifos exposure may have on the heart rate of children and adolescents using a cross-sectional study design.

\section{Methods}

Data from the 2001 to 2002 National Health and Nutrition Examination Survey was used in the analysis. These years were selected because they mark the timepoint when the residential use of chlorpyrifos was banned due to health concerns. Since the 1960 's, NHANES has been collecting surveys and performing examinations of US citizens to illustrate the overall health of the nation's people [21]. NHANES includes variables representing dietary intake, physical exams, laboratory reports, dental health, illnesses, medications, and sociodemographic data.

In total, there were 1252 children between the ages of 6 to 18 inclusive with TCPy data available in NHANES. This number was further reduced with the exclusion of missing heart rate data, and the removal of one outlier $(n=1233)$. Inclusion in this study was primarily based on urinary evaluation for pesticides available in the 2001-2002 NHANES Current Use Pesticide data subset. Other inclusion criteria focused on availability of data for either brachial or radial pulse rate, as well as associated covariates such as age, gender, and ethnicity.

The outlier was a six-year-old with a heart rate of 156 BPM which was 6 standard deviations above the recorded mean for that age in the sample ( $M=87.57$ BPM, $S D=11.28 \mathrm{BPM}$ ). This subject was excluded from the primary analysis with the assumption that the heart rate was recorded incorrectly. A post hoc sensitivity analysis was also performed to determine the influence of this outlier.

Heart rate was measured by a physician as radial pulse for children ages 6 and 7, whereas for subjects 8 and older, brachial pulse was assessed [22]. The pulse rate was measured over 30 seconds and doubled to obtain the number of beats per minute. Because the pulse rate approximates the heart rate, both pulse measures were combined in a single, complete variable for ease of analysis.

Chlorpyrifos can be metabolized into urinary 3,5,6-trichloropyridinol (TCPy) and excreted in urine, serving as an indicator of chlorpyrifos exposure [7]. TCPy concentrations were measured using capillary gas chromatography with tandem mass spectrometry (GC-MS/MS) [22]. The limit of detection was defined as 0.4 $\mu \mathrm{g} / \mathrm{L}$. For levels below the limit of detection (LOD), values were assigned by tak- 
ing the LOD divided by the square root of two (LOD/ $\sqrt{2})$ [22]. Thus, the imputed TCPy value under the LOD was given as $0.28 \mu \mathrm{g} / \mathrm{L}$. TCPy concentrations were reported in $\mu \mathrm{g} / \mathrm{L}$ units.

The NHANES 2001-2002 dataset was downloaded from the Centers for Disease Control website. The available subject data was matched on pulse, TCPy, age, gender, and ethnicity variables. A dichotomous TCPy variable was included in the dataset to denote if a child tested above the LOD or below it. Normality of data was assessed using the Shapiro-Wilk test. Because the data was not normally distributed, the pulse rate was natural log-transformed to improve normality of the distributions. Independent samples $t$-tests were performed to determine if there was a significant difference in pulse between subjects above or below the detection limit.

The $t$-test sample was stratified by age and gender due to a priori expectations that these variables could differentially affect heart rate outcomes. Because of diminished sample sizes when stratified by age, the sample was split into age groupings of 6 - 11 years old children $(n=539)$ and $12-18$ years old adolescents $(n=694)$. Participants were grouped based on NHANES examination age which varied depending on when they were initially interviewed versus when samples were collected for laboratory analysis. The numbers of participants varied because of the size and the non-normal age distribution included in the 2001-2002 NHANES Current Use Pesticide data subset. Using these groupings, a $t$-test was performed to test equality of means for pulse based on TCPy detection status. The geometric mean was evaluated for the continuous pulse rate and TCPy variables, as has been done in other studies.

Ordinary least squares regression was performed by regressing pulse rate onto natural log-transformed TCPy concentration. Age, gender, and ethnicity were input as covariates, with numerically coded values assigned to gender and ethnicity categorical variables. Separate regression models stratified by age groupings and gender were also created. Since previous studies indicated a differential effect with males and females, a regression model with an interaction term was constructed. Natural log-transformed TCPy concentrations below the LOD were centered at 0 for testing the interaction. Unstandardized regression coefficients were back-transformed through exponentiation. All analyses were conducted using SAS (version 9.4).

\section{Results}

\subsection{Descriptive Statistics}

There were 1233 total participants in the sample. The average age of the subjects was 12 years $(M=12.14, S D=3.70)$. The mean pulse was recorded as $79.69 \mathrm{BPM}$ $(S D=12.59)$ for the entirety of the sample. For the $6-11$ years grouping, the mean pulse was $83.64 \mathrm{BPM}(S D=11.27)$, and for $12-18$ years it was $76.63 \mathrm{BPM}$ $(S D=12.71) .47 \%(n=579)$ of the sample was male and $53 \%(n=654)$ was female. 401 (33\%) individuals were non-Hispanic black, 362 (29\%) were Mexican 
American, 358 (29\%) were non-Hispanic white, 59 (5\%) were other Hispanic, and $53(4 \%)$ were other.

TCPy values ranged from the imputed nondetectable level of $0.28 \mu \mathrm{g} / \mathrm{L}$ to the maximum detected level of $79.59 \mu \mathrm{g} / \mathrm{L}(M=5.15, S D=6.86)$. The mean TCPy value for detected subjects was $5.90 \mu \mathrm{g} / \mathrm{L}(S D=7.08)$. The majority of the sample tested positive for the TCPy biomarker 1068 (87\%), whereas only 165 (13\%) individuals were nondetects.

\subsection{Analysis}

After performing all analyses, no significant relationship between detection status and heart rate was found. The $t$-tests performed on these data indicated that the difference in pulse rate between detects and nondetects was nonsignificant overall and within each stratification (Table 1). Girls with urinary TCPy detections displayed a nonsignificant slower average heart rate than girls under the LOD (Figure 1). While nonsignificant, boys with urinary TCPy detections consistently demonstrated slightly elevated pulse rates compared to boys under the LOD (Figure 2). For adolescents ages $12-18\left(G M_{\text {detect }}=75.44, G M_{\text {nondetect }}=\right.$ $76.57)$ and in the entire sample overall $\left(G M_{\text {detect }}=78.63, G M_{\text {nondetect }}=79.05\right)$ the pulse rate was slightly and nonsignificantly slower $(p=0.42$ and $p=0.69$, respectively). Equality of means was also tested at each age level; however, when subdivided by each individual age based on the condition of detection, small samples limited the analysis (Figure 3).

The adjusted multiple regression models did not demonstrate any remarkable effect of TCPy concentration on pulse rate (Table 2). In girls, there was a nonsignificant positive association between pulse rate and TCPy concentrations $(\beta=$ $0.54,95 \% \mathrm{CI}, 0.27$ to $1.09, p=0.09$ ). Similarly, in boys a nonsignificant positive

Table 1. Results of $t$-test comparing TCPy concentration detects and nondetects on geometric means of pulse rate.

\begin{tabular}{ccccccccc}
\hline & \multicolumn{9}{c}{ Detect } & \multicolumn{2}{c}{ Nondetect } \\
\hline & $\mathrm{N}$ & $\mathrm{GM}(95 \% \mathrm{CI})$ & $\mathrm{N}$ & $\mathrm{GM}(95 \% \mathrm{CI})$ & $t$ & $p$ \\
\hline Overall pulse rate & 1068 & 78.63 & $(77.88,79.39)$ & 165 & 79.05 & $(77.10,81.06)$ & -0.40 & 0.69 \\
Boys & 510 & 76.33 & $(75.20,77.47)$ & 69 & 75.35 & $(72.26,78.57)$ & 0.59 & 0.56 \\
Girls & 558 & 80.80 & $(79.84,81.77)$ & 96 & 81.83 & $(79.45,84.28)$ & -0.79 & 0.43 \\
Children & 467 & 82.95 & $(81.95,83.96)$ & 72 & 82.39 & $(79.48,85.40)$ & 0.39 & 0.69 \\
Boys & 220 & 80.86 & $(79.34,82.41)$ & 35 & 77.71 & $(73.57,82.09)$ & 1.50 & 0.13 \\
Girls & 247 & 84.85 & $(83.58,86.15)$ & 37 & 87.07 & $(83.52,90.77)$ & -1.21 & 0.23 \\
Adolescents & 601 & 75.44 & $(74.44,76.45)$ & 93 & 76.57 & $(74.02,79.20)$ & -0.80 & 0.42 \\
Boys & 290 & 73.06 & $(71.57,74.58)$ & 34 & 72.99 & $(68.43,77.86)$ & 0.03 & 0.98 \\
Girls & 311 & 77.72 & $(76.43,79.03)$ & 59 & 78.70 & $(75.78,81.74)$ & -0.60 & 0.55 \\
\hline
\end{tabular}

Note. $\mathrm{N}=$ sample size. $\mathrm{GM}=$ geometric mean. $\mathrm{CI}=$ confidence interval. $t=t$-test statistic. $p=p$-value. Pulse rate is given in beats per minute (BPM). Overall includes entire sample ages 6 - 18; Children are in ages 6 - 11; Adolescents are in ages $12-18$. 
Table 2. Adjusted linear regression coefficients of the association between transformed TCPy concentrations and pulse rate.

\begin{tabular}{ccccccccccc}
\hline & \multicolumn{3}{c}{ All youth $^{\mathrm{a}}(n=1233)$} & \multicolumn{2}{c}{ Boys $^{\mathrm{b}}(n=579)$} & \multicolumn{2}{c}{$\operatorname{Girls}^{\mathrm{b}}(n=654)$} \\
\hline & $\beta(95 \% \mathrm{CI})$ & $p$ & $p_{\text {interaction }}$ & $\beta(95 \% \mathrm{CI})$ & $p$ & $\beta(95 \% \mathrm{CI})$ & $p$ \\
\hline Overall pulse & 1.13 & $(0.51,2.51)$ & 0.76 & 0.21 & 1.21 & $(0.52,2.83)$ & 0.65 & 0.54 & $(0.27,1.09)$ & 0.09 \\
Children & 1.35 & $(0.46,3.97)$ & 0.59 & 0.44 & 1.46 & $(0.47,4.53)$ & 0.51 & 0.64 & $(0.23,1.80)$ & 0.40 \\
Adolescents & 1.01 & $(0.32,3.19)$ & 0.98 & 0.38 & 1.10 & $(0.33,3.71)$ & 0.88 & 0.47 & $(0.18,1.25)$ & 0.13
\end{tabular}

Note. $\mathrm{CI}=$ confidence interval. Overall includes ages 6 - 18; Children are in ages $6-11$; Adolescents are in ages 12 - 18. Regression coefficients represent unstandardized values. ${ }^{a}$ Models were adjusted for ethnicity, gender, age, interaction term [ $\operatorname{sex}^{*} \ln \left(\right.$ TCPy concentration)]. ${ }^{b}$ Models were adjusted for ethnicity, gender, age.

95.00

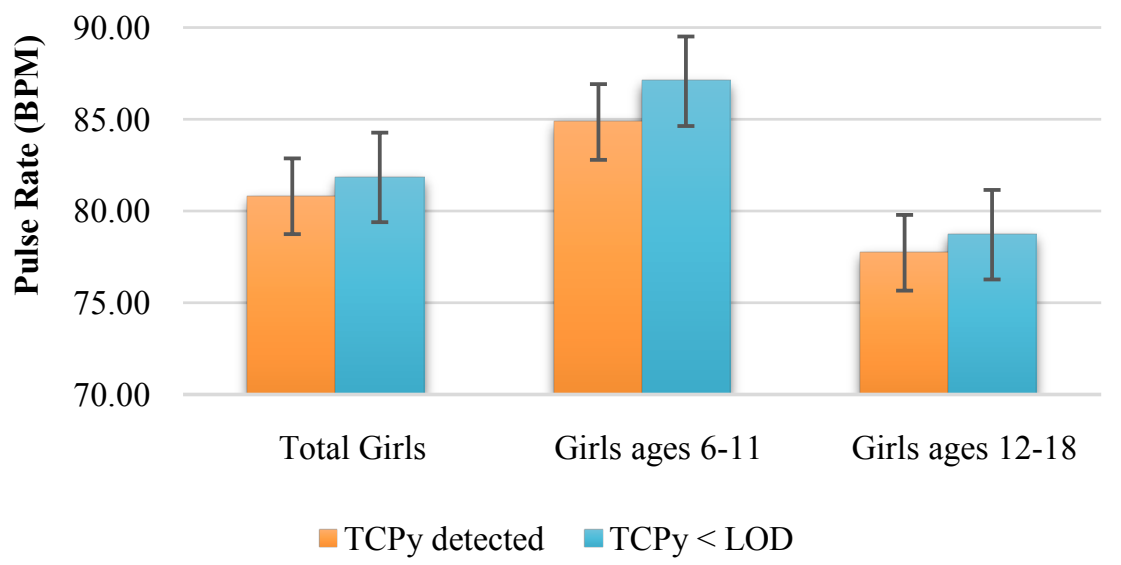

Figure 1. Comparison of mean pulse rates for detect versus nondetect in girls. Results of $t$-tests for equality of means among females who were either above or below the detection threshold. $\mathrm{GM}=$ geometric mean; $\mathrm{BPM}=$ beats per minute; $\mathrm{LOD}=$ limit of detection.

90.00

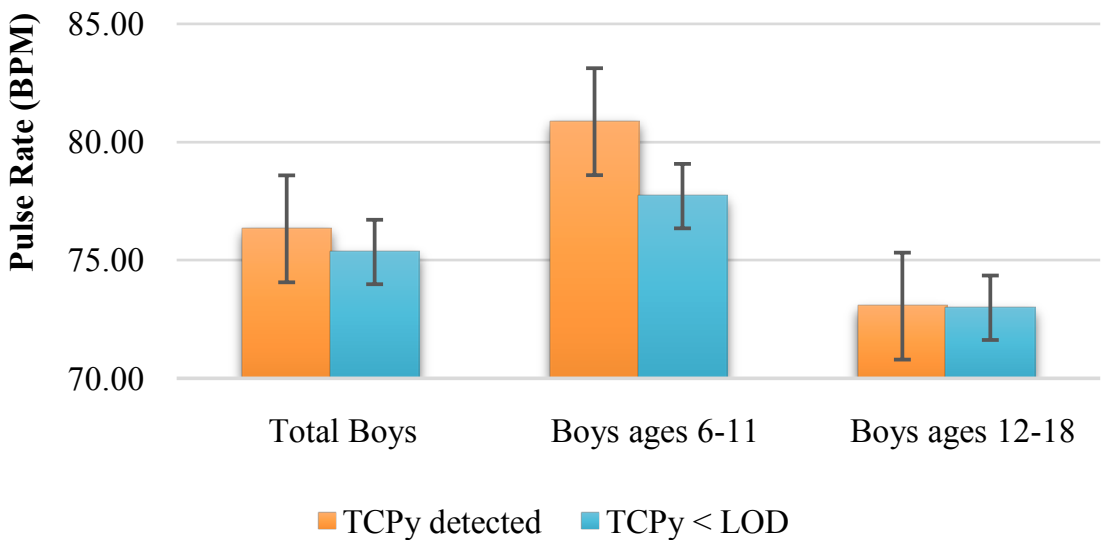

Figure 2. Comparison of mean pulse rates for detect versus nondetect in boys. Results of $t$-tests for equality of means among males who were either above or below the detection threshold. GM = geometric mean; $\mathrm{BPM}=$ beats per minute; $\mathrm{LOD}=$ limit of detection. 


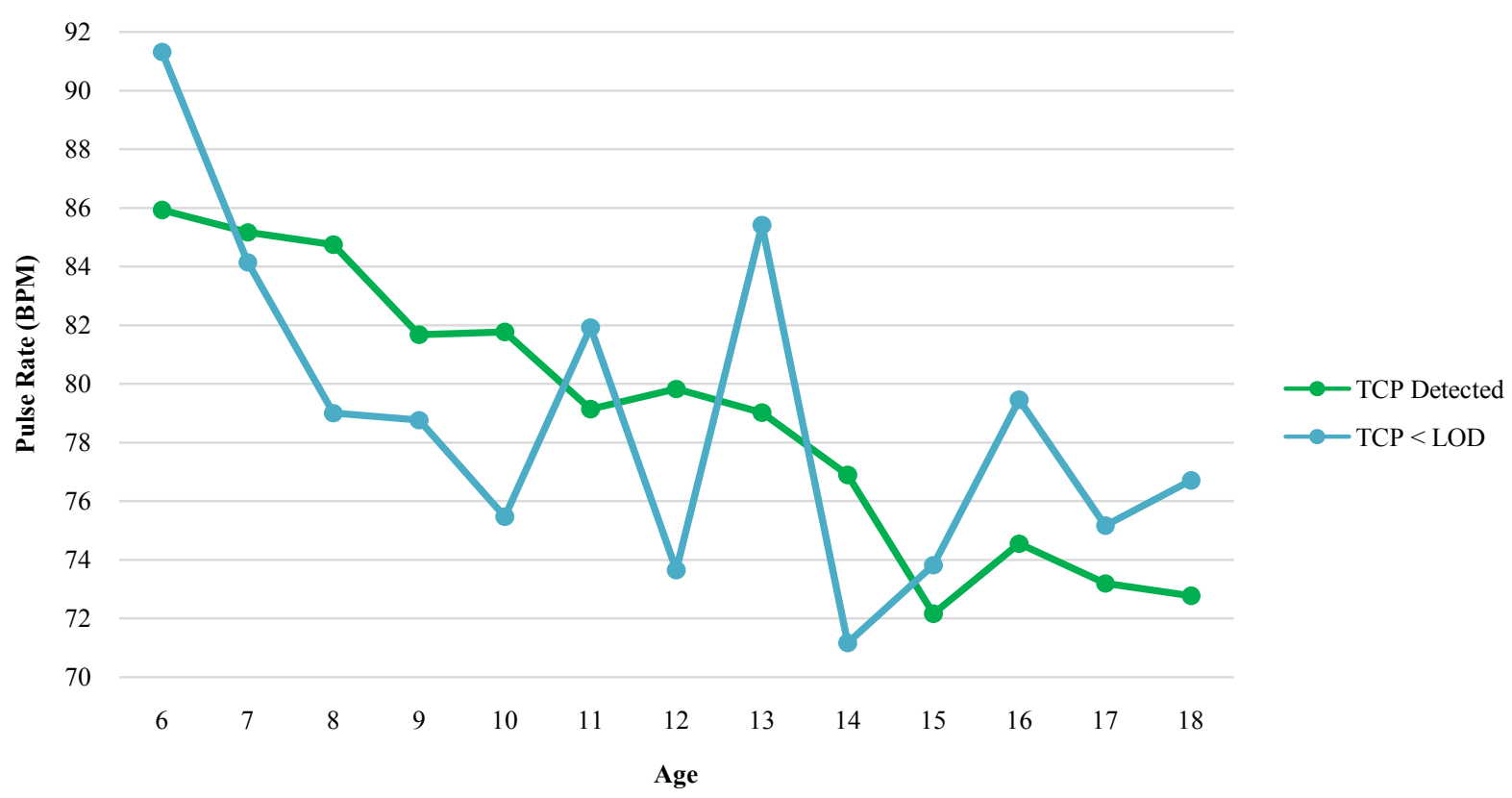

Figure 3. Comparison of mean pulse rates (GM) for detect versus nondetect by age. Results of $t$-tests for equality of means for the entire sample $(n=1,233)$ who were either above or below the detection threshold. Pulse rate in beats per minute is plotted against age. $\mathrm{GM}=$ geometric mean; $\mathrm{BPM}=$ beats per minute; $\mathrm{LOD}=$ limit of detection.

relationship between pulse and increasing TCPy metabolite levels was depicted ( $\beta=1.21,95 \% \mathrm{CI}, 0.52$ to $2.83, p=0.65)$. When stratified by age groupings, coefficients for boys and girls maintained this trend, representing a nonsignificant but positive trend.

Although there appeared to be differences in pulse rate between boys and girls, the subject's sex did not significantly impact the relationship between TCPy concentrations and pulse rate in the regression models. In Table 2, the sex and TCPy interaction term failed to demonstrate significance in the overall sample $(p=0.21)$, and when stratified by age groupings for children $(p=0.44)$ and adolescents $(p=0.38)$.

A post hoc sensitivity analysis was conducted to determine the impact of an extreme outlier which had a recorded heart rate of 156 BPM. The analysis was robust to the exclusion of the outlier, as the results of the OLS and $t$-tests did not reveal any apparent relationship between heart rate and TCPy metabolite concentrations.

\section{Discussion}

In this study, it was hypothesized that children with detectable concentrations of urinary TCPy metabolite would exhibit slower than average pulse rate. However, no significant effects on heart rate were discovered. Although there were subtle trends worth exploring: for example, the decreased heart rate in girls who detected as compared to girls below the LOD, and increased heart rate in boys who detected as compared to boys under the LOD.

Controlled laboratory studies on animal models and acute chlorpyrifos poi- 
soning events in humans have demonstrated mixed findings. In studies of acute chlorpyrifos poisoning in rats, doses of $25 \mathrm{mg} / \mathrm{kg}$ and $30 \mathrm{mg} / \mathrm{kg}$ have demonstrated bradycardia [20] [23]. However, doses of $10 \mathrm{mg} / \mathrm{kg}$ chlorpyrifos in the same rat strain did not elicit significant changes in heart rate [20]. A one-month duration of intraperitoneal chlorpyrifos injection of $7 \mathrm{mg} / \mathrm{kg}$ three times weekly in Wistar rats was sufficient to impair brainstem AChE levels significantly more than control values, although heart rate was unaltered [24]. Similarly, it was reported that post-natal day 1 rat pups heart rate was assessed as an indicator for chlorpyrifos-induced neurological effects [25]. The authors found that the PND1 pups in the treatment group received either $1 \mathrm{mg} / \mathrm{kg}$ chlorpyrifos or $2 \mathrm{mg} / \mathrm{kg}$ chlorpyrifos, but neither group displayed the anticipated bradycardic effects within two hours of chlorpyrifos injection [25].

In an attempt to evaluate the impact of probable OP exposure, researchers of the "ESPINA" study examined 4 - 9 years old children of floricultural workers. At the conclusion of the study, it was determined that there was no significant alteration in heart rate associated with $\mathrm{AChE}$ inactivation from OP pesticides [26]. However, it should be taken into account that environmental sampling was not conducted to determine if the timing of the study coincided with high or low periods of field pesticide application, or to identify the specific pesticides; rather, the investigators used educated assumptions on seasonal OP use. Furthermore, a surrogate measure of OP toxicity, AChE inhibition, was utilized to assess the exposure. Even with suspected exposure to AChE inhibitors, the lack of effect on heart rate from observational studies on humans and laboratory data is consistent with the findings in this paper.

The rationale of investigating child cardiovascular outcomes with chronic chlorpyrifos exposure is due to the reported neurological effects in children that chlorpyrifos may induce even at low levels. Through the same mechanism of AChE inhibition, chlorpyrifos has been implicated in inducing neurotoxicity within the central nervous system (CNS); however, regarding everyday exposures within the general population, this action is more controversial. A commonly-cited prospective study [27] reported that chlorpyrifos detected in mother's umbilical cord was inversely related to offspring Working Memory and Full Scale IQ when assessed at age 7. However, this has been disputed in a re-analysis of the data in which the results were not able to be replicated [28]. The author asserts that such low, subacute levels of chlorpyrifos would be incapable of causing neurological impairment because brain AChE would be unaffected [29]. However, other literature suggests that the manifestation of chlorpyrifos toxicity may be a result of other physiological mechanisms independent of chlorpyrifos-oxon binding to AChE [30]. Other studies have reported possible genotoxicity and oxidative damage at subtoxic doses administered either orally or subcutaneously in Wistar and SD rats [31] [32].

Unraveling the putative effects of subacute chlorpyrifos exposure as it relates to the CNS and cardiac functionality will require more in depth, longitudinal investigations. Furthermore, whether AChE inhibition is the sole mechanism 
behind chlorpyrifos toxicity or not remains to be determined. Future studies looking at chlorpyrifos and blood pressure are warranted, as the literature indicated a possible relationship between OP exposure and blood pressure, even at subacute levels. Mixed findings of both increases [33] and decreases [26] in systolic blood pressure have been reported due to usual, subtoxic exposure to general OP in humans.

Strengths of this analysis include the examination of real-life exposures to a common pesticide. However, it should be noted that due to NHANES sampling methods to increase minority representation, the proportions of ethnic groups in this sample may not be representative of the US population overall. Availability of data also substantially diminished sample sizes when stratifying by age. Despite these factors, there were still no significant findings.

With only TCPy metabolite data available, providing indisputable evidence of a correlation between chlorpyrifos exposure and associated cardiac outcomes is not possible. Furthermore, it was not ideal that two different methods of monitoring the pulse were used, depending upon the age of the child. Although these measures are used interchangeably, consistency would have been preferred. Due to the nature of cross-sectional studies, the variability in TCPy concentrations over time was not able to be captured. There was no way of determining the timing and fluctuations in exposure and if that was associated with any heart-related outcomes.

\section{Conclusion}

Findings in the current study were unable to demonstrate a significant effect of chlorpyrifos exposure on heart rate. No significant relationships between the TCPy biomarker and heart rate were observed on the sample overall, nor in the stratified groupings for age. There were still no significant findings when stratifying for sex and no indication that any differences between pulse rate among the sexes could be attributed to TCPy exposure. Neither was any effect modification of sex observable in the linear regression. The nonsignificant regression coefficient for TCPy indicates that chlorpyrifos exposure did not contribute to any of the explained variation in pulse rate in the model. Differential pulse rates in the population at the given chlorpyrifos exposure levels could not be demonstrated.

\section{Acknowledgements}

Supported in part by Center for Environmental and Occupational Risk Analysis and Management, College of Public Health, University of South Florida, Tampa, Florida.

\section{Conflicts of Interest}

The authors declare no conflicts of interest regarding the publication of this paper. 


\section{References}

[1] Sheppard, L., McGrew, S. and Fenske, R.A. (2020) Flawed Analysis of an Intentional Human Dosing Study and Its Impact on Chlorpyrifos Risk Assessments. Environment International, 143, Article ID: 105905. https://doi.org/10.1016/j.envint.2020.105905

[2] Ming, Y., Beach, J., Martin, J.W. and Senthilselvan, A. (2016) Urinary Dialkyl Phosphate Concentrations and Lung Function Parameters in Adolescents and Adults: Results from the Canadian Health Measures Survey. Environmental Health Perspectives, 124, 491-497. https://doi.org/10.1289/ehp.1509745

[3] United States Environmental Protection Agency [EPA] (2014) Chlorpyrifos: Revised Human Health Risk Assessment for Registration Review. DP Barcode D424485. https://beta.regulations.gov/document/EPA-HQ-OPP-2008-0850-0195

[4] Engel, S.M., Wetmur, J., Chen, J., Zhu, C., Barr, D.B., Canfield, R.L., et al. (2011) Prenatal Exposure to Organophosphates, Paraoxonase 1, and Cognitive Development in Childhood. Environmental Health Perspectives, 119, 1182-1188. https://doi.org/10.1289/ehp.1003183

[5] Howard, M., Mirajkar, N., Karanth, S. and Pope, C. (2007) Comparative Effects of Oral Chlorpyrifos Exposure on Cholinesterase Activity and Muscarinic Receptor Binding in Neonatal and Adult Rat Heart. Toxicology, 238, 157-165. https://doi.org/10.1016/j.tox.2007.05.030

[6] Davis, D.L. and Ahmed, A.K. (1998) Exposures from Indoor Spraying of Chlorpyrifos Pose Greater Health Risks to Children than Currently Estimated. Environmental Health Perspectives, 106, 299-301.

https://doi.org/10.1289/ehp.98106299

[7] Eaton, D.L., Daroff, R.B., Autrup, H., Bridges, J., Buffler, P., Costa, L.G., et al. (2008) Review of the Toxicology of Chlorpyrifos with an Emphasis on Human Exposure and Neurodevelopment. Critical Reviews in Toxicology, 38, 1-125.

https://doi.org/10.1080/10408440802272158

[8] United States Environmental Protection Agency [EPA] (2019) Chlorpyrifos. https://www.epa.gov/ingredients-used-pesticide-products/chlorpyrifos\#: :text=Chlorpyr ifos\%20is\%20an\%20organophosphate $\% 20$ insecticide,of $\% 20$ food $\% 20$ and $\% 20$ feed $\% 20 \mathrm{cr}$ ops

[9] D’Agostino, J., Zhang, H., Kenaan, C. and Hollenberg, P.F. (2015) Mechanism-Based Inactivation of Human Cytochrome P450 2B6 by Chlorpyrifos. Chemical Research in Toxicology, 28, 1484-1495. https://doi.org/10.1021/acs.chemrestox.5b00156

[10] Uwaifo, F. and John-Ohimai, F. (2020) Dangers of Organophosphate Pesticide Exposure to Human Health. Matrix Science Medica, 4, 27-31. https://doi.org/10.4103/MTSM.MTSM_2_20

[11] Marsillach, J., Costa, L.G. and Furlong, C.E. (2016) Paraoxonase-1 and Early-Life Environmental Exposures. Annals of Global Health, 82, 100-110. https://doi.org/10.1016/j.aogh.2016.01.009

[12] Keifer, M.C. and Firestone, J. (2007) Neurotoxicity of Pesticides. Journal of Agromedicine, 12, 17-25. https://doi.org/10.1300/J096v12n01_03

[13] Eskenazi, B., Kogut, K., Huen, K., Harley, K.G., Bouchard, M., Bradman, A., et al. (2014) Organophosphate Pesticide Exposure, PON1, and Neurodevelopment in School-Age Children from the CHAMACOS Study. Environmental Research, 134, 149-157. https://doi.org/10.1016/j.envres.2014.07.001 
[14] Saulsbury, M.D., Heyliger, S.O., Wang, K. and Round, D. (2008) Characterization of Chlorpyrifos-Induced Apoptosis in Placental Cells. Toxicology, 244, 98-110. https://doi.org/10.1016/j.tox.2007.10.020

[15] Whyatt, R.M., Camann, D., Perera, F.P., Rauh, V.A., Tang, D., Kinney, P.L., et al. (2005) Biomarkers in Assessing Residential Insecticide Exposures during Pregnancy and Effects on Fetal Growth. Toxicology and Applied Pharmacology, 206, 246-254. https://doi.org/10.1016/j.taap.2004.11.027

[16] Huen, K., Harley, K., Bradman, A., Eskenazi, B. and Holland, N. (2010) Longitudinal Changes in PON1 Enzymatic Activities in Mexican-American Mothers and Children with different genotypes and Haplotypes. Toxicology and Applied Pharmacology, 244, 181-189. https://doi.org/10.1016/j.taap.2009.12.031

[17] Roy, A., Guatimosim, S., Prado, V.F., Gros, R. and Prado, M.A.M. (2014) Cholinergic Activity as a New Target in Diseases of the Heart. Molecular Medicine, 20, 527-537. https://doi.org/10.2119/molmed.2014.00125

[18] Davies, J., Roberts, D., Eyer, P., Buckley, N. and Eddleston, M. (2008) Hypotension in Severe Dimethoate Self-Poisoning. Clinical Toxicology, 46, 880-884.

https://doi.org/10.1080/15563650802172063

[19] Anand, S., Singh, S., Nahar Saikia, U., Bhalla, A., Paul Sharma, Y. and Singh, D. (2009) Cardiac Abnormalities in Acute Organophosphate Poisoning. Clinical Toxicology, 47, 230-235. https://doi.org/10.1080/15563650902724813

[20] Gordon, C.J. and Padnos, B.K. (2000) Prolonged Elevation in Blood Pressure in the Unrestrained Rat Exposed to Chlorpyrifos. Toxicology, 146, 1-13.

https://doi.org/10.1016/S0300-483X(00)00158-X

[21] Centers for Disease Control and Prevention [CDC]. About NHANES: Introduction. 2017. https://www.cdc.gov/nchs/nhanes/about_nhanes.htm

[22] National Center for Health Statistics [NCHS] (2002) National Health and Nutrition Examination Survey [Data File and Codebook]. 2001-2002. NCHS, Maryland.

[23] Felippe, I.S.A., Müller, C.J.T., Siqueira, A.A., Dos Santos, L., Cavadino, A., Paton, J.F.R., et al. (2020) The Antidotes Atropine and Pralidoxime Distinctively Recover Cardiorespiratory Components Impaired by Acute Poisoning with Chlorpyrifos in Rats. Toxicology and Applied Pharmacology, 389, Article ID: 114879. https://doi.org/10.1016/j.taap.2020.114879

[24] Batista, T.J., Minassa, V.S., Aitken, A.V., Jara, B.T., Felippe, I.S.A., Beijamini, V., et al. (2019) Intermittent Exposure to Chlorpyrifos Differentially Impacts Neuroreflex Control of Cardiorespiratory Function in Rats. Cardiovascular Toxicology, 19, 548-564. https://doi.org/10.1007/s12012-019-09528-7

[25] Bourgeois, M., Driver, J., Ross, J. and Harbison, R. (2019) Evaluation of Postnatal Chlorpyrifos Exposure on Heart Rate of PND1 Rats. American Chemical Society Fall 2019 National Meeting and Expo, Medical Toxicology Section, San Diego, CA, August 2019, 100.

[26] Suarez-Lopez, J.R., Jacobs Jr., D.R., Himes, J.H. and Alexander, B.H. (2013) Acetylcholinesterase Activity, Cohabitation with Floricultural Workers, and Blood Pressure in Ecuadorian Children. Environmental Health Perspectives, 121, 619-624. https://doi.org/10.1289/ehp.1205431

[27] Rauh, V., Arunajadai, S., Horton, M., Perera, F., Hoepner, L., Barr, D.B. and Whyatt, R. (2011). Seven-Year Neurodevelopmental Scores and Prenatal Exposure to Chlorpyrifos, a Common Agricultural Pesticide. Environmental Health Perspectives, 119, 1196-1201. https://doi.org/10.1289/ehp.1003160

[28] Dourson, M., Gadagbui, B., Onyema, C. and McGinnis, P. (2020) A Commentary 
on Some Epidemiology Data for Chlorpyrifos. Regulatory Toxicology and Pharmacology, 113, Article ID: 104616. https://doi.org/10.1016/j.yrtph.2020.104616

[29] Marty, M.S., Andrus, A.K., Bell, M.P., Passage, J.K., Perala, A.W., Brzak K.A., et al. (2012) Cholinesterase Inhibition and Toxicokinetics in Immature and Adult Rats after Acute or Repeated Exposures to Chlorpyrifos or Chlorpyrifos-Oxon. Regulatory Toxicology and Pharmacology, 63, 209-224. https://doi.org/10.1016/j.yrtph.2012.03.015

[30] Abreu-Villaça, Y. and Levin, E.D. (2017) Developmental Neurotoxicity of Succeeding Generations of Insecticides. Environment International, 99, 55-77. https://doi.org/10.1016/j.envint.2016.11.019

[31] Muller, M., Hess, L., Tardivo, A., Lajmanovich, R., Attademo, A., Poletta, G., et al. (2014) Neurologic Dysfunction and Genotoxicity Induced by Low Levels of Chlorpyrifos. Neurotoxicology, 45, 22-30. https://doi.org/10.1016/j.neuro.2014.08.012

[32] Kopjar, N., Žunec, S., Mendaš, G., Micek, V., Kašuba, V., Mikolić, A., et al. (2018) Evaluation of Chlorpyrifos Toxicity through a 28-Day Study: Cholinesterase Activity, Oxidative Stress Responses, Parent Compound/Metabolite Levels, and Primary DNA Damage in Blood and Brain Tissue of Adult Male Wistar Rats. Chemico-Biological Interactions, 279, 51-63. https://doi.org/10.1016/j.cbi.2017.10.029

[33] Harari, R., Julvez, J., Murata, K., Barr, D., Bellinger, D.C., Debes, F., et al. (2010) Neurobehavioral Deficits and Increased Blood Pressure in School-Age Children Prenatally Exposed to Pesticides. Environmental Health Perspectives, 118, 890-896. https://doi.org/10.1289/ehp.0901582 